\title{
Molecular Structure of an Icosahedral Pyruvate Dehydrogenase Complex
}

Sriram Subramaniam*, Dan Shi*, Richard N. Perham**, and Jacqueline L. S. Milne***,

* Laboratory of Biochemistry, National Cancer Institute, NIH, Bethesda, MD USA 20892

** University of Cambridge, Cambridge CB2 1GA, UK.

***Laboratory of Cell Biology, National Cancer Institute, NIH, Bethesda, MD USA 20892

Complex cellular processes such as signal transduction, gene expression, motility and energy metabolism are often implemented using multi-component molecular assemblies. Knowledge of the architecture of these molecular machines is central to an understanding of their mechanisms. Some large complexes such as the nucleosome, the ribosome and the proteasome have been successfully analyzed by X-ray crystallographic techniques. Structures of other assemblies, such as the family of 2-oxo acid dehydrogenase multienzyme complexes, have proved impossible to obtain by crystallographic methods, in part because of their large size but also because of the intrinsic structural heterogeneity introduced by the mobile domains and "swinging arms" that are essential to transfer catalytic intermediates between the active sites of their constituent enzymes.

Like the PDH complexes of eukaryotes and of other Gram-positive bacteria, the $B$. stearothermophilus PDH is assembled around a core of 60 dihydrolipoyl acetyltransferase (E2) chains arranged with icosahedral symmetry. Each E2 chain consists of three domains: (i) an $\mathrm{N}$-terminal $9 \mathrm{kDa}$ lipoyl domain, which visits the active sites of the pyruvate decarboxylase (E1) and then those of E2 and dihydrolipoyl dehydrogenase (E3), (ii) a $4 \mathrm{kDa}$ peripheral subunit-binding domain (PSBD) to which E1 and E3 bind tightly and mutually exclusively, and (iii) a C-terminal $28 \mathrm{kDa}$ catalytic (acetyltransferase) domain, 60 copies of assemble to form the icosahedral inner core. These domains are linked by stretches of extended, conformationally flexible polypeptide chain.

We have recently arrived at three-dimensional models for (i) the 1.8 megadalton icosahedral complex composed of sixty copies of the E2 catalytic domain (at a resolution of $14.5 \AA$ ), and (ii) an 11 megadalton, icosahedral PDH complex composed of sixty copies each of E1 and E2 (at a resolution of $28 \AA$ ). Atomic interpretations of each of these complexes were obtained by combining the electron microscopy-derived structures with previously determined atomic coordinates of the individual components of the complexes. Analysis of the models provides a number of novel insights into the design and function of this molecular machine. A key feature is that the E1 molecules are located on the periphery in an orientation that allows each of the 60 mobile lipoyl domains tethered to the inner E2 enzyme to access multiple E1 active sites from inside the icosahedral complex. This unanticipated architecture provides a highly efficient mechanism for active site coupling and catalytic rate enhancement, which we propose is achieved by the motion of the lipoyl domain in the restricted annular region between the inner and outer cores of the complex. 


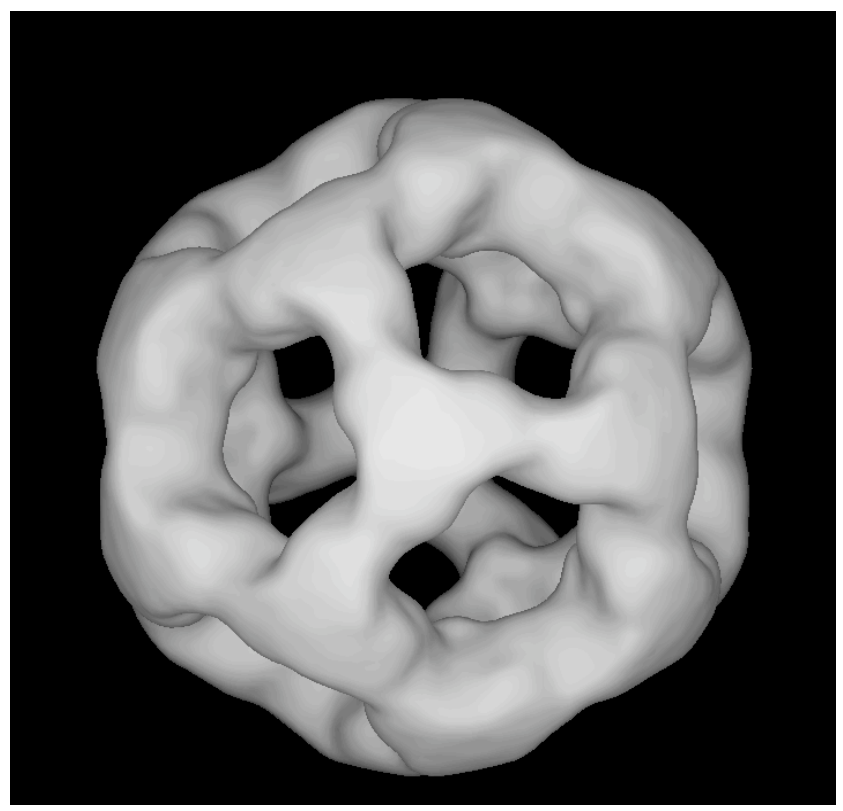

Fig. 1. Surface representation of the icosahedral complex formed by the E2 catalytic domain. The complex has a diameter of about $225 \AA$.

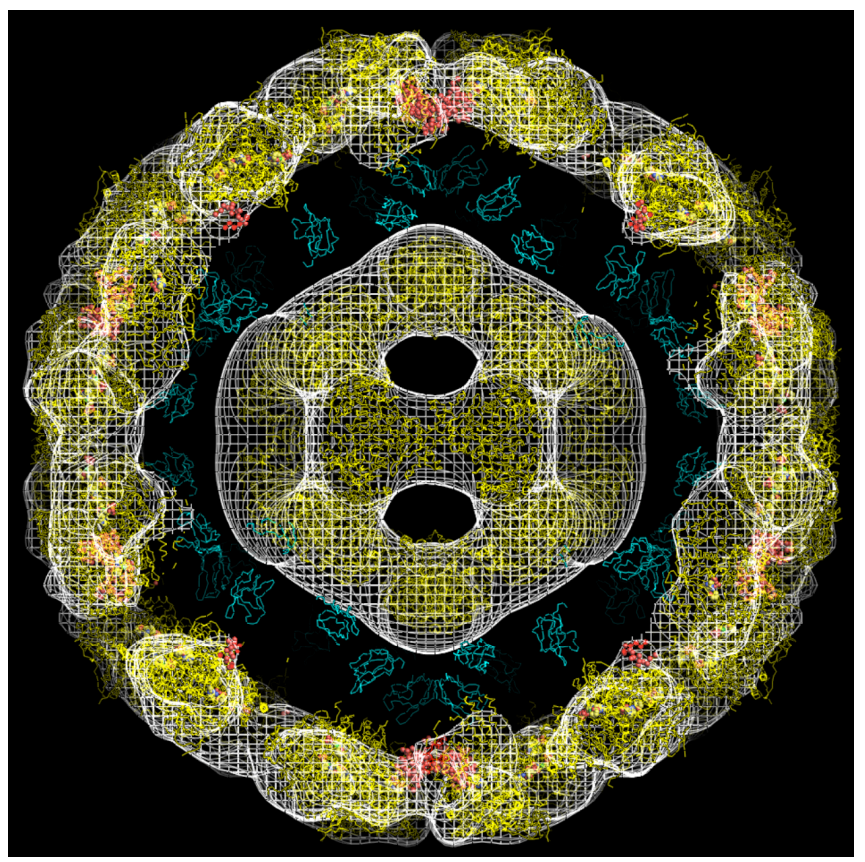

Fig. 2. A cross-sectional view of the E1E2 complex displaying the probable locations of the three domains of the E2 chain and the E1 tetramer. The complex has a diameter of about $475 \AA$. The E1 tetramers and the E2 binding domain (in red) are on the periphery, the E2 catalytic domain is at the core, and E2 lipoyl domain is in the annular region. 